\title{
Un atelier implicatif : « je suis unique »
}

\section{Mondher Abrougui et Pierre Clément}

\section{(2) OpenEdition}

Journals

Édition électronique

URL : http://journals.openedition.org/trema/2092

DOI : 10.4000/trema.2092

ISSN : 2107-0997

\section{Éditeur}

Faculté d'Éducation de l'université de Montpellier

\section{Édition imprimée}

Date de publication : 1 septembre 1996

Pagination : 177-181

ISSN : 1167-315X

Référence électronique

Mondher Abrougui et Pierre Clément, « Un atelier implicatif : « je suis unique » », Tréma [En ligne],

9-10 | 1996, mis en ligne le 18 septembre 2013, consulté le 04 mai 2019. URL : http://

journals.openedition.org/trema/2092 ; DOI : 10.4000/trema.2092

Ce document a été généré automatiquement le 4 mai 2019.

Trema 


\title{
Un atelier implicatif : " je suis unique»
}

\author{
Mondher Abrougui et Pierre Clément
}

\section{Introduction}

1 L'atelier que nous avons animé aux Rencontres de Montpellier, a été initialement inspiré par une recherche sur l'îlot «Fais ta carte d'identité » de la Cité des Enfants à la Cité des Sciences et de l'Industrie, La Villette, Paris (Abrougui, 1994 ; Clément et al, 1995 ; Abrougui et Clément, sous presse).

2 La génétique n'est introduite dans les programmes scolaires qu'en classe de troisième. Or les enfants et leurs parents se posent une foule de questions sur l'hérédité, et plusieurs ouvrages ou revues de vulgarisation (notamment Jacquard 1978, 1982, 1983) ont commencé à répondre à ces questions, ainsi que des expositions (Langaney et al, 1992). L'initiative de la Cité des Enfants d'introduire les termes de "caractères génétiques ", " caractères héréditaires », « unique ", « sosie », etc. dans l'îlot « Fais ta carte d'identité " entre dans le cadre de cette éducation non formelle, que les parents et les pédagogues peuvent utiliser pour pallier l'absence de traitement de ces questions dans un contexte strictement scolaire. Cet îlot aborde des notions de génétique particulièrement délicates, car marquées aussi par des enjeux sociaux, voire idéologiques, et par des savoirs communs profondément ancrés (dès sa naissance, le bébé est comparé au grand-père, à la mère, etc.). Les objectifs et propos de cet îlot sont limités : faire remplir une «carte d'identité biologique ", à partir de l'identification de caractères qui ne changent jamais (couleur des cheveux, couleur de la peau, couleur des yeux, aspect des empreintes digitales, aptitude à rouler la langue et forme du lobe de l'oreille) et d'autres qui changent avec le temps ou l'âge (taille, poids, ...). Le message est que chacun d'entre nous a une combinaison unique de caractères.

3 L'atelier que nous avons proposé, inspiré de cet îlot, a été testé à plusieurs reprises dans un contexte scolaire, avec tous les enfants d'une ou plusieurs classes, mais initialement pour seulement les 6 caractères génétiques à partir desquels les enfants réalisent leur propre carte d'identité à la Cité des Enfants (cf. ci-dessus). 
Dans l'atelier que nous avons animé à Montpellier, nous avons privilégié plus de critères de différentiation, avec les objectifs suivants :

- introduire des notions, biologiques ou non, à partir desquelles il est possible de réfléchir à la question «qu'est-ce qui peut constituer mon identité, la caractériser ?»

- montrer ainsi qu'il y a de multiples voies qui permettent d'affirmer que je suis unique, ce qui révèle que l'approche actuellement privilégiée par les manuels scolaires (limitée à l'identité génétique) n'est qu'un point de vue auquel ne peut pas se résumer ce qui fait mon originalité, mon identité.

5 Les caractères génétiques sont-ils ou non les seuls caractères immuables qui permettent de répondre à la question «suis-je unique?»? Et sont-ils les plus pertinents pour répondre à cette question?

6 Comment à travers une telle activité introduire ou aborder avec des élèves le problème de la diversité et du polymorphisme?

\section{Déroulement de l'atelier}

7 Nous avons d'abord rassemblé les participants en un groupe unique, puis nous l'avons partitionné en sous-groupes de plus en plus petits, selon des critères annoncés verbalement par un des animateurs, et récapitulés au tableau par l'autre animateur. En fin de partition, chaque sous-groupe n'était plus constitué que d'un seul membre, parfois de deux.

8 Cette démarche est mise en œuvre deux fois. Lors de la première fois, les critères de partition des groupes étaient tous des caractères génétiques immuables (listés cidessous). Lors de la seconde fois, les critères de partition étaient tous des caractères non génétiques mais cependant immuables eux aussi (listés également ci-dessous).

Notre objectif n'étant pas uniquement d'obtenir des individus uniques, mais surtout de permettre une réflexion sur les critères de classification et d'appartenance à des groupes différents. À l'issue de chacune des deux partitions du groupe initial, chaque participant était invité à consigner par écrit, et de façon anonyme, ses propres conceptions sur les causes du constat auquel aboutissait le jeu collectif: « je suis unique (ou presque)». À la fin de chaque activité nous avons donc posé à tous les participants la même question: "êtes-vous unique? Expliquer ce qui fait que vous êtes ou non unique?"

liste des caractères génétiques qui ont été utilisés pour la première partition du groupe

- Yeux, clairs ou sombres ;

- Langue, (aptitude à la rouler ou non) ;

- Lobe de l'oreille, collé ou non ;

- Petit ou grand, $(<$ ou > 1,75 m) ;

- Groupe sanguin, (O ou autre) ;

- Cheveux, (couleur initiale avant teinture éventuelle : clairs, foncés ou roux)

- Cheveux lisses ou non lisses, (ondulés, frisés) ;

- Peau, claire ou mate ;

- Ça s'est arrêté là, mais on avait aussi en réserve :

- Le type d'empreintes digitales ;

- Le rhésus sanguin + ou - ;

- etc. 
11 Liste des caractères non génétiques mais immuables qui ont été utilisés pour la seconde partition du groupe

- Aimer manger épicé ou non ;

- Année de naissance paire ou impaire ;

- Mois de naissance comportant 31 jours ou moins de 31 ;

- Jour de naissance, (avant le 10, entre le 11-20, après le 21 du mois);

- Age $<30$ ans ; 30 à 40 ans ; et $>40$ ans ;

- Lieu de naissance au dessus ou au dessous du $45^{\mathrm{e}}$ parallèle ;

- Ça s est arrêté là, mais on avait aussi en réserve :

- Département de naissance, côtier ou continental ;

- Nom qui commence par une voyelle ou par une consonne ;

- Prénom qui commence par une voyelle ou par une consonne ;

- Port de lunettes ou non (rien ou lentilles) ;

- Signe astrologique.

12 Il est évident que la liste peut être très variée, mais elle est toutefois suffissante pour permettre une réflexion sur la notion d'unicité.

\section{Analyse des réponses récoltées lors de l'atelier}

13 Nous avons, bien après l'atelier, regroupé les réponses écrites des participants selon les catégories suivantes:

- Génétique : cause uniquement génétique (allèles, gènes, brassage génétique, mutations)

- Probabilité : argumentation basée sur les probabilités (combinaison, chances, brassage)

- Histoire et expérience personnelle : référence à l'histoire ou expérience individuelle

- Reproduction sexuée : cause due à la reproduction sexuée

- Socioculturel : cause due à des facteurs socioculturels (éducation, culture, etc)

- Complexité du vivant : causes multiples, liées à la complexité du vivant

\subsection{Après la première activité}

Tous les participants étaient uniques et leurs explications écrites sont résumées dans le tableau 1.

\subsection{Après la deuxième activité}

15 Parmi les 18 participants, 14 étaient uniques et 4 étaient non uniques; leurs explications écrites sont résumées dans le même tableau 1 : 


\begin{tabular}{|c|c|}
\hline Après la première activité & Après la deuxième activité \\
\hline Participants tous uniques : & Participants uniques : \\
\hline 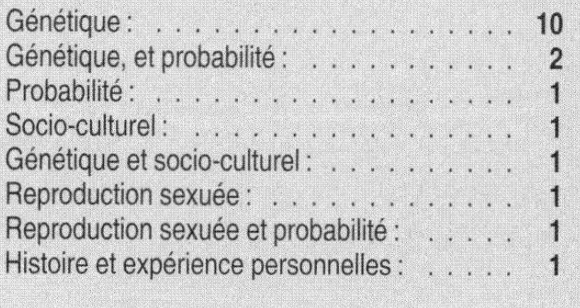 & 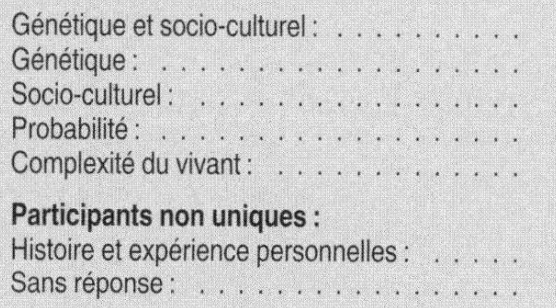 \\
\hline
\end{tabular}

\section{Discussion et conclusions}

Au total, le déroulement de l'atelier et de la discussion qui a suivi nous ont convaincu
qu'un tel jeu, s'il est reproduit à l'avenir par les uns ou les autres, dans un cadre scolaire
ou extrascolaire, gagnerait à différencier dans la deuxième phase (la phase sur les critères
non génétiques), les paramètres civils immuables qui constituent mon identité civile
(nom et prénom, date et lieu de naissance, etc.) des paramètres socioculturels qui
constituent mon identité à la suite de mon histoire individuelle unique : peu de questions
évoquaient cette dimension, en dehors de la première sur les goûts culinaires; toute
question sur les lieux fréquentés par chacun au cours de sa vie, les musiques entendues
ou préférées, les préférences vestimentaires ou de parfums, les connaissances et capacités

Au total, le déroulement de l'atelier et de la discussion qui a suivi nous ont convaincu
qu'un tel jeu, s'il est reproduit à l'avenir par les uns ou les autres, dans un cadre scolaire
ou extrascolaire, gagnerait à différencier dans la deuxième phase (la phase sur les critères
non génétiques), les paramètres civils immuables qui constituent mon identité civile
(nom et prénom, date et lieu de naissance, etc.) des paramètres socioculturels qui
constituent mon identité à la suite de mon histoire individuelle unique : peu de questions
évoquaient cette dimension, en dehors de la première sur les goûts culinaires; toute
question sur les lieux fréquentés par chacun au cours de sa vie, les musiques entendues
ou préférées, les préférences vestimentaires ou de parfums, les connaissances et capacités

Au total, le déroulement de l'atelier et de la discussion qui a suivi nous ont convaincu
qu'un tel jeu, s'il est reproduit à l'avenir par les uns ou les autres, dans un cadre scolaire
ou extrascolaire, gagnerait à différencier dans la deuxième phase (la phase sur les critères
non génétiques), les paramètres civils immuables qui constituent mon identité civile
(nom et prénom, date et lieu de naissance, etc.) des paramètres socioculturels qui
constituent mon identité à la suite de mon histoire individuelle unique : peu de questions
évoquaient cette dimension, en dehors de la première sur les goûts culinaires; toute
question sur les lieux fréquentés par chacun au cours de sa vie, les musiques entendues
ou préférées, les préférences vestimentaires ou de parfums, les connaissances et capacités

Au total, le déroulement de l'atelier et de la discussion qui a suivi nous ont convaincu
qu'un tel jeu, s'il est reproduit à l'avenir par les uns ou les autres, dans un cadre scolaire
ou extrascolaire, gagnerait à différencier dans la deuxième phase (la phase sur les critères
non génétiques), les paramètres civils immuables qui constituent mon identité civile
(nom et prénom, date et lieu de naissance, etc.) des paramètres socioculturels qui
constituent mon identité à la suite de mon histoire individuelle unique : peu de questions
évoquaient cette dimension, en dehors de la première sur les goûts culinaires; toute
question sur les lieux fréquentés par chacun au cours de sa vie, les musiques entendues
ou préférées, les préférences vestimentaires ou de parfums, les connaissances et capacités

Au total, le déroulement de l'atelier et de la discussion qui a suivi nous ont convaincu
qu'un tel jeu, s'il est reproduit à l'avenir par les uns ou les autres, dans un cadre scolaire
ou extrascolaire, gagnerait à différencier dans la deuxième phase (la phase sur les critères
non génétiques), les paramètres civils immuables qui constituent mon identité civile
(nom et prénom, date et lieu de naissance, etc.) des paramètres socioculturels qui
constituent mon identité à la suite de mon histoire individuelle unique : peu de questions
évoquaient cette dimension, en dehors de la première sur les goûts culinaires; toute
question sur les lieux fréquentés par chacun au cours de sa vie, les musiques entendues
ou préférées, les préférences vestimentaires ou de parfums, les connaissances et capacités

Au total, le déroulement de l'atelier et de la discussion qui a suivi nous ont convaincu
qu'un tel jeu, s'il est reproduit à l'avenir par les uns ou les autres, dans un cadre scolaire
ou extrascolaire, gagnerait à différencier dans la deuxième phase (la phase sur les critères
non génétiques), les paramètres civils immuables qui constituent mon identité civile
(nom et prénom, date et lieu de naissance, etc.) des paramètres socioculturels qui
constituent mon identité à la suite de mon histoire individuelle unique : peu de questions
évoquaient cette dimension, en dehors de la première sur les goûts culinaires; toute
question sur les lieux fréquentés par chacun au cours de sa vie, les musiques entendues
ou préférées, les préférences vestimentaires ou de parfums, les connaissances et capacités

Au total, le déroulement de l'atelier et de la discussion qui a suivi nous ont convaincu
qu'un tel jeu, s'il est reproduit à l'avenir par les uns ou les autres, dans un cadre scolaire
ou extrascolaire, gagnerait à différencier dans la deuxième phase (la phase sur les critères
non génétiques), les paramètres civils immuables qui constituent mon identité civile
(nom et prénom, date et lieu de naissance, etc.) des paramètres socioculturels qui
constituent mon identité à la suite de mon histoire individuelle unique : peu de questions
évoquaient cette dimension, en dehors de la première sur les goûts culinaires; toute
question sur les lieux fréquentés par chacun au cours de sa vie, les musiques entendues
ou préférées, les préférences vestimentaires ou de parfums, les connaissances et capacités

Au total, le déroulement de l'atelier et de la discussion qui a suivi nous ont convaincu
qu'un tel jeu, s'il est reproduit à l'avenir par les uns ou les autres, dans un cadre scolaire
ou extrascolaire, gagnerait à différencier dans la deuxième phase (la phase sur les critères
non génétiques), les paramètres civils immuables qui constituent mon identité civile
(nom et prénom, date et lieu de naissance, etc.) des paramètres socioculturels qui
constituent mon identité à la suite de mon histoire individuelle unique : peu de questions
évoquaient cette dimension, en dehors de la première sur les goûts culinaires; toute
question sur les lieux fréquentés par chacun au cours de sa vie, les musiques entendues
ou préférées, les préférences vestimentaires ou de parfums, les connaissances et capacités chercheurs en didactique de la biologie) avaient souvent des opinions bien arrêtées que le seul jeu pratiqué ne suffisait pas à faire évoluer. Les changements d'argumentation des réponses écrites ont en effet été moins massivement importants que ce que la structure des deux jeux successifs laissait a priori espérer.

fréquence de l'argumentation génétique (je suis unique parce que mon génome est unique), présente dans 13 réponses sur 18 après le jeu sur les critères génétiques, mais encore clans 7 réponses sur 18 après le jeu sur les critères autres que génétiques.

évoqué dans ce type de situation (atelier de didactique de la biologie), alors que ce sont les paramètres les plus utilisés socialement: le moindre relevé d'identité comporte des questions sur le nom et prénom de chacun, sur ses date et lieu de naissance, et éventuellement sur le nom des parents: l'ensemble est suffisant pour identifier quiconque.

évoquée, qui n'a pas d'emblée le sérieux de «l'originalité de chaque génome humain sauf pour les jumeaux homozygotes». Or nombre de traits de la personnalité de chacun n'expriment que l'originalité de leur histoire (depuis le langage jusqu'aux goûts, en passant par les connaissances, les capacités à résoudre certains problèmes, etc.).

Tréma, 9-10 | 2010 
acquises, etc. mérite alors d'être posée et peut être tout aussi discriminante que les questions sur les caractères génétiques ou civils.

\section{RÉSUMÉS}

Un jeu a permis de regrouper les participants de l'atelier en ensembles de plus en plus réduits jusqu'à ne comporter qu'un individu (parfois deux), qui est donc unique par rapport aux autres, eu égards aux critères utilisés pour scinder successivement le groupe. Dans un premier jeu, ces critères ont été des phénotypes à déterminisme génétique strict. Dans un deuxième jeu, ils ont porté sur des paramètres socioculturels ou liés à l'état civil.

La discussion et l'évaluation écrite ont permis de montrer que ces différents types de paramètres n'ont pas le même statut aux yeux des participants, certains étant considérés comme plus sérieux que d'autres. Il conviendrait à l'avenir de distinguer trois types d'identité : génétique, civile et socioculturelle.

By means of a game, it was shown to be possible to split the participants of the workshop into smaller and smaller units up to the point where some units had only one member, (sometimes two), who were then unique in relation to the other participants, as regards the criteria used to split the group. In the first game these criteria were phenotypes of a strictly deterministic genetic kind. In a second game, they were socio cultural parameters linked to the legal identity of the members.

Through the discussion and the written evaluation, it was shown that these different types of parameters are not of equal importance in the participants' view. Some are considered more serious than others. In the future, it would be advisable to distinguish three types of criteria: genetic, legal, and socio-cultural.

\section{INDEX}

Mots-clés : didactique, génétique, identité civile, identité génétique, identité socioculturelle, jeu Keywords : didactic, genetic, genetic identity, legal identity, play, socio-cultural identity

\section{AUTEURS}

\section{MONDHER ABROUGUI}

Equipe de recherche en didactique de la biologie, LIRDHIST, université Claude Bernard de Lyon

\section{PIERRE CLÉMENT}

Equipe de recherche en didactique de la biologie, LIRDHIST, université Claude Bernard de Lyon 